\title{
Oral cancer and precancer screening studies have high discriminatory ability
}

Moles DR, Downer MC, Speight PM. Meta-analysis of measures of performance reported in oral cancer and precancer screening studies. Br Dent J 2002; 192:340-344

Objective Systematic review and meta-analysis of the performance of screening measures for oral cancer and precancer.

Data sources PubMed (Medline), SCISEARCH and the Cochrane Library up to December 2000, hand-searching of relevant journals, and discussions with relevant authors.

Study selection Prospective field studies which related specifically to validation of oral cancer or precancer screening using at least a 'soft' gold-standard. Studies estimating parameters of screening performance, response to screening invitation and compliance in follow-up.

Data extraction and synthesis Global estimates for sensitivity and specificity were obtained from the selected studies using the summary receiver operator characteristic (SROC) curve meta-analytical technique.

Results Six studies were included, and weighted pooled averages for sensitivity, specificity and likelihood ratios were calculated (See Table 1).

Conclusions There was considerable heterogeneity with respect to location, population and screening personnel in the studies but this had only a moderate effect on sensitivity and specificity. The
Table 1 Calculated weighted pooled average for sensitivity and the corresponding value for specificity and likelihood ratios.

\begin{tabular}{lcc}
\hline & Pooled average & 95\% Confidence intervals \\
\hline Sensitivity & 0.796 & $0.594-0.912$ \\
Specificity & 0.977 & $0.941-0.991$ \\
Likelihood ratio & 35 & $14-89$ \\
\hline
\end{tabular}

discriminatory ability demonstrated by the screening personnel, irrespective of their grade and training, was generally of a fairly high order.

Evidence-Based Dentistry (2002) 3, 79-80. doi:10.1038/ sj.ebd.6400136

Address for reprints: Dr DR Moles, Department of Oral Pathology, Eastman Dental Institute for Oral Health Care Sciences, University College London, 256 Gray's Inn Road, London WC1X 8LD, UK.

E-mail: d.moles@eastman.ucl.ac.uk.

\section{Commentary}

This meta-analysis addresses an important health issue and an area where quality research is lacking: the discriminatory ability of visual oral cancer screenings. A meta-analysis is a statistical method for combining the results from a number of similar studies to produce an overall estimate of effect or summary measure from results included. The meta-analysis consisted of sensitivity values but, in addition, prevalence rates, specificity, and positive and negative predictive values based on visual examinations of the oral mucosa from six studies capturing seven oral cancer and precancer screening programmes, were also reported.

The a priori establishment of inclusion and exclusion criteria, an essential component of meta-analysis, allows for selection of similar studies that can be combined. Nevertheless, there is heterogeneity even in similar studies. Moles et al. provide a cursory overview of the heterogeneity of the six studies included in their meta-analysis, stating that the oral cancer screening programmes had different types of examiners, calibration, disease prevalence in the population and threshold for a positive finding. The latter two have a direct and potentially pronounced effect on sensitivity and specificity and the authors provide a clear and concise explanation of this concept. Unfortunately, a detailed description of the varied circumstances in which these studies were conducted is missing. It is important to note, however, that the considerable heterogeneity only moderately influenced the sensitivity and specificity values.

The authors discuss one limitation of the study in that a biopsy was not required as the gold standard for a positive outcome. Instead, a 'soft' gold standard, that is, detailed examination by an oral medicine or surgeon specialist, was considered acceptable. This limitation is further compounded because the authors do not specify in which studies, if any, a precancerous lesion was considered a positive outcome, based solely on a visual examination. Given this 'soft' gold standard and the fact that suspicious lesions often prove to be benign upon biopsy, it is probable that there were more false-positives than reported. Furthermore, it has been demonstrated that between 4.5 and $15.3 \%$ of precancers and early-stage oral cancers cannot be adequately identified by visual inspection alone and may be overlooked even by highly trained professionals, potentially increasing the falsenegative rate. ${ }^{1}$ 
The authors provide a thoughtful, albeit brief, overview of the difficulties in conducting clinical trials of oral cancer screenings. Their novel approach to meta-analysis, which utilised the results of screening tests, gave rise to the pooled-weighted sensitivity value of 0.796 and a corresponding specificity value of 0.977 (95\% confidence interval, 0.941$0.991)$. When specificity was held at 0.977 , the corresponding value of sensitivity is 0.796 ( $95 \%$ confidence interval, 0.594-0.912). These results suggest that these oral cancer screening studies have a high discriminatory ability regardless of the examiners qualifications or screening methods employed, however, in our opinion, the article contains inadequate information to fully evaluate this conclusion.

1. Sciubba JJ. Improving detection of precancerous and cancerous oral lesions. J Am Dent Assoc 1999; 130:1445-1457.

Paul S Farsai Department of General Dentistry, Boston University Goldman School of Dental Medicine, Boston, Massachusetts, USA

Michelle Henshaw Department of Health Policy \& Health Service Research, Boston University Goldman School of Dental Medicine, Boston, Massachusetts, USA

\section{Further Discussion - Editor}

As we endeavour where possible to allow the original author to see the commentary on their article prior to publication this leads to interesting interchanges. In this particular case a number of points were raised which highlight both the difficulties of commentary writing on meta-analysis published in peer-review journals and the challenges facing the commentary writers in producing objective comments.

The commentary raises the failure to deal with sources of heterogeneity between the studies. Here the authors of the paper were to a considerable extent the victims of the need to limit the length of a manuscript intended for publication in a peer-reviewed journal. However, this issue is an inevitable circumstance of submitting manuscripts for publication, and the commentators have a clear and valid point. This is because in reading a metaanalysis, it is very important for the reader and practitioner to understand the scope and variety of circumstances in which the described studies were conducted. The simple solution to this is for complete or fuller details of the material and methods to be published, as may be found in for example Cochrane reviews. Another alternative which some journals e.g. British Medical Journal and Canadian Dental Journal have employed is to provide additional material on their website. This allows the interested reader further access. The more traditional approach is for the interested reader to read the original papers. If we are to see better reporting of systematic reviews along the lines of the QUOROM statement ${ }^{1}$ perhaps more journals should look to either increasing their word limits for systematic review or look at the electronic route.

The issue of the 'gold standard' is important. There is no doubt that histological findings from biopsy would, in principle, be a greatly superior validating criterion to expert clinical examination. By incorporating a 'soft' standard, the doors are opened for screening 'abnormal' lesions, not necessarily cancer or pre-cancer lesions, which were supposed to be the purpose of the study. So using a 'soft' gold standard gives the unavoidable likelihood of misclassification in both directions.

There is a dilemma here, for based on this article alone readers as the commentators are unable to ascertain a clear definition of a 'positive' outcome in the article. This is because of two issues, the lack of clarity regarding positive outcomes in the original studies and the lack of a 'hard' gold standard. Furthermore if the undoubted 'gold standard' of histology was a necessary condition to include studies into the meta-analysis it is highly doubtful whether there would have been anything to report, and this is a useful review of the area. Finally on the topic of the 'gold standard' there is the question of whether it would be ethically acceptable to subject apparently healthy mucosa to biopsy. For in order to determine the true sensitivity and specificity for oral examination ALL patients (or at least to a random selection of the negatives) who screened negative or positive would have to be subjected to biopsy in order to establish the correct diagnosis. While applying the 'gold standard to only those who screen positive seems attractive, it introduces a verification bias.

It is also important to stress that we still as yet know very little about the natural history of oral cancer and potentially malignant lesions (PMLs). Inventories of these PMLs were included in most of the included papers but the lists were not identical. This is a further cause of heterogeneity in the review and also for potential screening, or more realistically case-finding for oral cancer and PMLs.

The commentators agree with the authors that the results suggest high discriminatory ability for oral cancer screening. However, and this is where a difference lies, they feel that because pieces of information are not fully reported as highlighted in the discussion above they feel that there is not enough information to fully evaluate the conclusion. The commentators role is to bring this to readers attention, the ultimate decision as to whether they follow the authors or the commentators is the readers decisions. This is the evidence-based approach in action.

1. Moher D, Cook DJ, Eastwood S, Olkin I, Rennie D, Stroup DF. Improving the quality of reports of meta-analyses of randomized controlled trials: the QUOROM statement. Lancet 1999; 354:1896-1899.

Derek Richards Editor 